\title{
A COMPUTER SIMULATION OF THE NON-NEWTONIAN BLOOD FLOW AT THE AORTIC BIFURCATION
}

\author{
ZHENG LOU* and WEN-JEI YANG $\dagger$ \\ *Transportation Research Institute, University of Michigan, Ann Arbor, MI 48109, U.S.A. and \\ †Mechanical Engineering and Applied Mechanics, University of Michigan, Ann Arbor, MI 48109, U.S.A.

\begin{abstract}
A two-dimensional numerical model was developed to determine the effect of the non-Newtonian behavior of blood on a pulsatile flow at the aortic bifurcation. The blood rheology was described by a weak-form Casson equation. The successive-over-relaxation (SOR) method was used to solve both the vorticity and Poisson equations numerically. It was disclosed that the non-Newtonian property of blood did not drastically change the flow patterns, but caused an appreciable increase in the shear stresses and a slightly higher resistance to both flow separations and the phase shifts between flow layers.
\end{abstract}

\section{NOMENCLATURE}

$\mathrm{AR}$
$D$
$D_{\mathrm{l}}$
$H$
$J, \bar{J}$ and $J^{\prime}$
$L$
$L_{\mathrm{b} \min }$
$n$
$R e$
$R e_{\mathrm{pt}}$
$r_{\mathrm{i}}$
$r_{\mathrm{o}}$
$s$

V

$x, y$

$x$

$\chi, \bar{x}$ and $\alpha^{\prime}$

$\beta$

$\beta, \bar{\beta}$ and $\beta^{\prime}$

$\therefore \bar{\gamma}$ and $\gamma^{\prime}$

$\mu$

$\xi, \eta$

$\sigma, \bar{\sigma}$ and $\sigma^{\prime}$

$\tau, \bar{\tau}$ and $\tau^{\prime}$

$\tau$

$\tau_{0}$

$\tau_{y}$

$\bar{\tau}_{y}$ and $\tau_{y}^{\prime}$

$\omega$

Subscripts

$\begin{array}{ll}\mathrm{b} & \text { branch } \\ \mathrm{t} & \text { trunk } \\ \mathrm{y} & \text { yield }\end{array}$

Received in final form 9 June 1992

\section{INTRODUCTION}

area ratio

diameter

second invariant of the strain rate tensor half-width of a channel

coordinate transformation coefficients

reference length

minimum branch length

parameter in the Papanastasiou's weak form

Reynolds number

peak Reynolds number in the trunk

transition curve radius at the vertex

transition curve radius at the hip

dimensionless displacement along a wall, with the origin being at the vertex for the inner wall and at the hip for the outer wall

time

reference velocity

spatial domain

Womersley number

coordinate transformation coefficients

bifurcation angle

coordinate transformation coefficients

coordinate transformation coefficients

shear rate

Casson viscosity or limiting apparent viscosity

effective or apparent viscosity

kinematic viscosity

referential domain

density

coordinate transformation coefficients

coordinate transformation coefficients

shear stress

time constant for a starting flow

yield shear stress

dimensionless yield shear stresses

dimensionless viscosity function

stream function

cardiac frequency or vorticity

trunk
The curvatures, junctions and bifurcations of large and medium arteries are severely affected by atherosclerosis. It has been suggested that fluid dynamics may play a role in the genesis and progression of the disease. The blood flows in these regions are disturbed and the arterial walls are exposed to either high or low shear stresses. Many experimental and theoretical studies have been conducted to investigate the role of fluid dynamics in atherogenesis. Comprehensive reviews pertinent to the subject are available (Lou, 1990; Nerem and Levesque, 1987). The involvement of the fluid dynamics in atherogenesis is subtle; however, various studies favor the association of a low shear region with atherosclerosis (Nerem and Levesque, 1987).

The current study is to reveal the effect of blood rheology on the fluid dynamics and the consequent implications in atherogenesis at the aortic bifurcation. It is commonly believed that the non-Newtonian effect is small in large vessels, where the shear rate is high. However, low shear spots also exist near bends and bifurcations. The current study uses the Casson model, which is known to adequately represent nonNewtonian blood rheology (Charm and Kurland, 1965). Liepsch and coworkers (Liepsch, 1985, 1986; Liepsch and McMillan, 1986; Liepsch and Moravec. 1984; Moravec and Liepsch, 1983) studied flows in renal and general T-shaped bifurcations with a power-law non-Newtonian fluid. Perktold et al. (1989) studied a Y-shaped bifurcation with an aneurysm in the cerebrovascular system with the Casson model. More recently, Perktold et al. (1991a) studied a carotid bifurcation with the Casson model. The Casson model, like many other models, does not address a time-dependent thixotropic behavior that blood shows. There have been no studies using the non-Newtonian models on the aortic bifurcation, which is where the abdominal aorta is divided into two iliac arteries. 
The present study employs a two-dimensional model that is tractable computationally although the related physiological situation is three-dimensional. There have been some three-dimensional simulations for the T-shaped (Perktold and Peter, 1990), carotid (Perktold et al., 1991 a, b; Rindt et al., 1990) and aortic (Yung et al., 1990; Wille, 1984) bifurcations. Wille (1984) used a nonphysiological Reynolds number of 10, while Yung et al. (1990) selected a nonphysiological area ratio of $\mathbf{2 . 0}$ to produce flow geometry for a convenient mesh generation. In addition, both Wille (1984) and Yung et al. (1990) applied steady flow.

For the carotid bifurcation, a two-dimensional model can give basic information on the complexity of the flow field, but it cannot take into account the important secondary flow that can be seen in a threedimensional model (Perktold et al., 1991b). The deviation induced by the secondary flow is found in the more distal parts (van de Vosse et al., 1990; Perktold et $a l ., 1991 \mathrm{~b}$ ). In the case of the aortic bifurcation, secondary flows are not observed in the branches with the area ratios of 0.8 and 1.2 and a bifurcation angle of $70^{\circ}$ during pulsatile flow, although they are observed during steady flow (Walburn and Stein, 1982). In another study of the aortic bifurcation with a bifureation angle of $91^{\circ}$ and an area ratio of 0.96 , the secondary flow was found both in steady and pulsatile flow (Fukushima et al., 1988). However, the region affected by the secondary flow is smaller during pulsatile flow than during steady flow. There were other studies that found the secondary flow with a quasi-steady flow rate, which has either a low frequency or a large ratio of the mean flow over the peak flow (Walburn and Stein, 1982). The current model is similar to that of Walburn and Stein (1982) and will only cause small error, if any. In a recent study (Hearshen, 1991), the results from the same two-dimensional model, but with a Newtonian fluid, showed good agreement with those from magnetic resonance measurements for pulsatile flows, and some deviations for steady flows.

\section{PHYSICAL MODEL}

\section{Geometry}

The flow is in channels of infinite depth (Fig. 1). $H_{\mathrm{t}}$ and $H_{\mathrm{b}}$ are the half-widths of the trunk and branch, respectively, while $\beta$ is the bifurcation angle. The vertex is the tip of the flow divider, while the hip is at the turning point of the outer wall. Let $r_{i}$ and $r_{0}$ be the radii of the transition curves at the vertex and the hip, respectively. The system geometry is symmetrical with respect to the trunk centerline and the flow rates in the two branches are equal. Therefore, the analysis needs to be performed on only one-half of the system. The actual aortic bifurcation may not be symmetric, and the effect of asymmetry on the flow is also an important subject (Friedman et al., 1983).

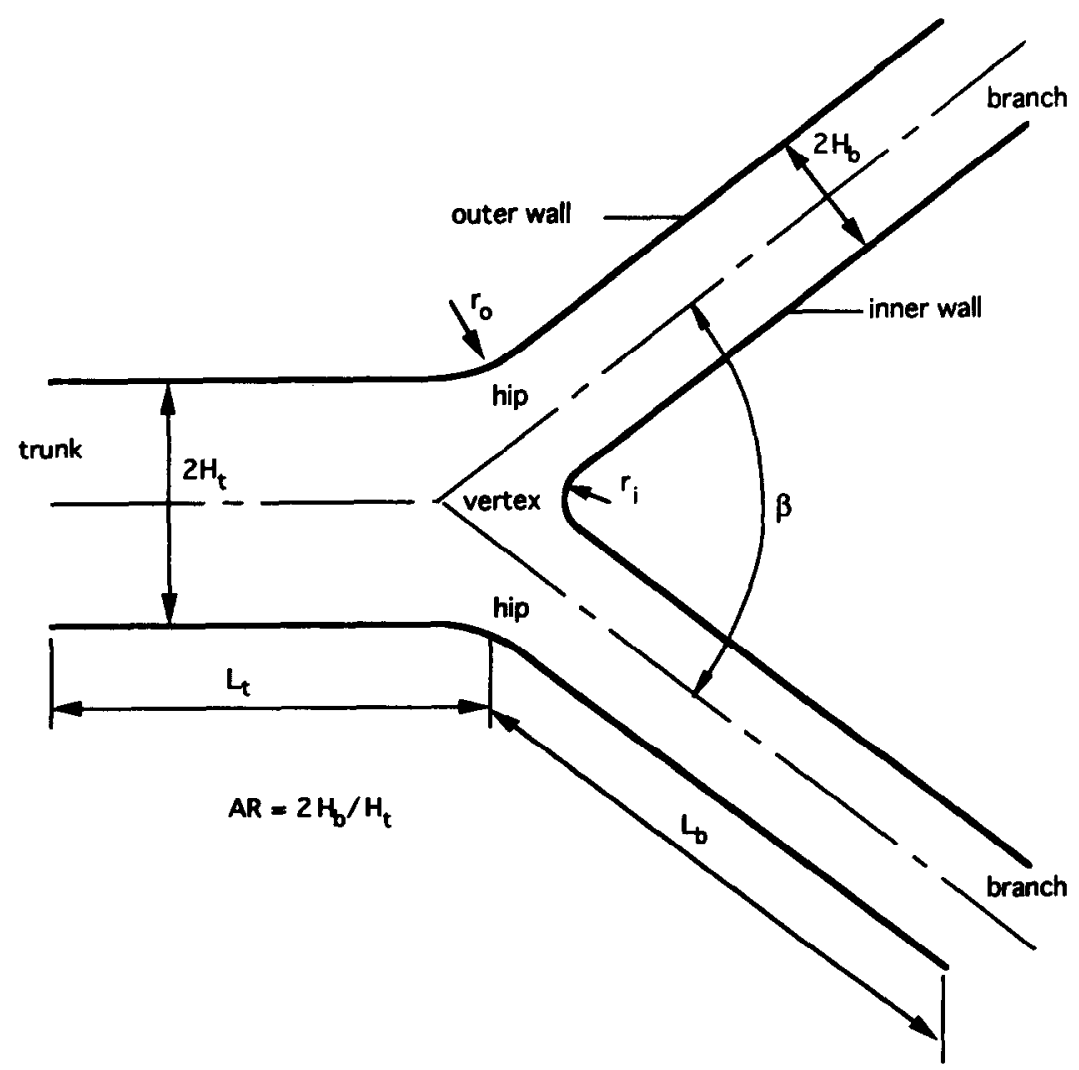

Fig. 1. A schematic diagram of a two-dimensional aortic bifurcation. The channels are of infinite depth. 




Phase angle (degrees)

Fig. 2. Physiologically pulsatile flow rate over one cycle proximal to the aortic bifurcation (adapted from Stettler et al., 1981). The total cycle lasts for $1 \mathrm{~s}$ in a normal case.

A peak Reynolds number is defined in the trunk. with the flow rate peaking at peak 1 (Fig. 2), as

$$
R e_{\mathrm{pt}}=L V / v,
$$

where $L$ is the characteristic length, equal to the trunk hydrodynamic diameter $\left(4 \mathrm{H}_{\mathrm{t}}\right)$, chosen to be $15 \mathrm{~mm}$, $V$ the characteristic velocity, equal to the average velocity across a hydrodynamically equivalent circular pipe, and $v$ the kinematic viscosity, equal to $4.5 \times 10^{-6} \mathrm{~m}^{2} \mathrm{~s}^{-1} . R e_{\mathrm{pt}}$ is equal to 1300 . The timeaverage Reynolds number over one cardiac cycle is 181, a laminar flow. The area ratio, AR, is defined as the ratio of the total area of two branches over the trunk area, and its values are 0.75 and 1.1 .

Murray (1926) observed that the bifurcation angle varies from 60 to $75^{\circ}$; Walburn et al. (1979) found this angle to be between 37 and $80^{\circ}$; and Friedman et al. (1981) observed this angle to be approximately $51^{\circ}$. Feuerstein et al. (1976) adopted a bifurcation angle of $75^{\circ}$, which is also the case in the present study. However, the present authors have studied the effects of the angle variation from 40 to $90^{\circ}$ (Lou and Yang, 1991)

Both the trunk and branches are long enough to avoid the end effects. The trunk length $L_{1}$ is equal to $1.2 L$, and the minimum branch length $L_{\mathrm{b} \text { min }}$ is calculated using the following equation (Patil and Subbaraj, 1988);

$$
L_{\mathrm{b} \text { min }} / L=0.004 \mathrm{AR} \times R e_{\mathrm{pt}} .
$$

Most of the previous numerical studies used sharp corners at the vertex and hip. In reality, the transitions are smooth. Goldman (1981) used a round corner as the flow divider, with no detailed information about the flow field around the corner. The authors have studied the effect of corner curvatures (Lou and Yang, 1991). In the present study, however, their values are fixed with $r_{i}=0.15 L$ and $r_{0}=0.2 L$.

\section{The Womersley parameter}

The Womersley number, $\alpha$, is an important parameter in pulsatile flows. It is defined as

$$
\alpha=\frac{D}{2} \sqrt{\frac{\omega}{v}} \text { for a circular pipe, }
$$

where $D$ is the pipe diameter and $\omega$ is the frequency. The equation can also be expressed as (Lou, 1990)

$$
\alpha^{2}=36.336 \frac{\tau_{0}}{T},
$$

where $\tau_{0}$ is the time constant for a starting flow in the pipe, and $T$ the period of the oscillating flow. $\tau_{0}$ reflects the influences from inertia and viscous forces. For a channel flow to be comparable with that in a circular pipe, the same equation should hold. With the time constant for a starting channel flow equal to $H^{2} / 1.571^{2} v$, one gets

$$
\alpha=1.531 \mathrm{H} \sqrt{\frac{\bar{\omega}}{v}} \text { for a channel, }
$$

where $H$ is $H_{t}$ for the current study. The trunk Womersley number is 8.8623 for a heart rate of 60 beats $\min ^{-1}$.

\section{The Casson model}

The Casson equation was originally proposed for shear thinning fluids, such as printer ink (Casson, 1959), with its stress-strain-rate relation

$$
\tau^{1 / 2}=\eta^{1 / 2} \dot{\gamma}^{1 / 2}+\tau_{\mathrm{y}}^{1 / 2},
$$

where $\tau_{y}$ is the yield shear stress, $\dot{\gamma}$ the shear rate, and $\eta$ the Casson viscosity or the asymptotic apparent viscosity, which is calculated from

$$
\eta=\rho v,
$$

where $\rho$ is the density, and $v$ the kinematic viscosity in an equivalent Newtonian fluid. The peak Reynolds number in the trunk is defined as

$$
R e_{\mathrm{pl}}=\rho L V / \eta \text {. }
$$

The Casson equation is valid for blood viscosity over a wide range of cell concentrations and shear rates of $1100,000 \mathrm{~s}^{-1}$ (Charm and Kurland, 1962, 1965) or 0.1-20 s ${ }^{-1}$ (Merril et al., 1964; Cokelet et al., 1963). For shear rates less than $1 \mathrm{~s}^{-1}$, shear stress data deviate from the Casson equation (Merril et al., 1966). The existence of yield stress remains a controversial problem (Lightfoot, 1974). At a higher shear rate of over $100 \mathrm{~s}^{-1}$ (Hussain, 1977) or $50 \mathrm{~s}^{-1}$ (Charm and Kurland, 1972), blood behaves like a Newtonian fluid obeying the relationship $\tau=\eta \dot{\gamma}$.

An effective or apparent viscosity for the Casson model is defined as

$$
\mu=\frac{\tau}{\dot{\gamma}}=\frac{\left(\sqrt{\eta} \sqrt{\dot{\gamma}}+\sqrt{\tau_{y}}\right)^{2}}{\dot{\gamma}} .
$$

Here $\mu$ is singular as $\dot{\gamma}$ approaches zero. Adopting a method used to overcome a similar singularity in a Bingham plastic fluid (Papanastasiou, 1987), one 
gets the following weak form of the Casson equation

$$
\mu=\frac{\left[\sqrt{\eta} \sqrt{\dot{\gamma}}+\sqrt{\tau_{y}}\left(1-\mathrm{e}^{-n|\dot{y}|}\right)\right]^{2}}{\dot{\gamma}},
$$

where $n$ takes a finite value.

\section{Yield shear stresses}

Merril et al. $(1965,1966)$ showed that fibrinogen behaves like a glue in a reversible aggregation of erythrocytes, that the yield shear stress, $\tau_{y}$, increases with the concentration of fibrinogen and the hematocrit composition, and that other plasma proteins have no effect in the absence of fibrinogen. However, an in vivo study of dog blood indicates that factors other than fibrinogen may be involved (Frasher et al., 1968). Some values of $\tau_{y}$ available in the literature are listed in Table 1.

The Newtonian, Bingham, Casson and weak Cas-

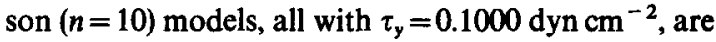
compared (Fig. 3). The deviation between the Bingham and Casson models is appreciable. The weak Casson model has no shear stress jump at zero shear rate, and it is almost identical to the Casson model for the shear rates exceeding $0.4 \mathrm{~s}^{-1}$.

\section{MATHEMATICAL FORMULATIONS}

The Casson equation is sufficient to analyze Poiseuille and Couette flows, for which the shear stress and strain distributions are known from statics and kinematics. A constitutive equation is necessary to solve more complicated problems, such as a bifurcation flow. The constitutive relation for a Casson fluid is (Fung, 1981)

$$
\mu\left(D_{\mathrm{II}}\right)=\frac{\left(\sqrt{\eta} \sqrt{\left.\sqrt{2 D_{\mathrm{II}}}+\sqrt{\tau_{\mathrm{y}}}\right)^{2}}\right.}{\sqrt{2 D_{\mathrm{II}}}},
$$

where $D_{\pi}$ is the second invariant of the strain rate tensor. The $\sqrt{2 D_{\mathrm{II}}}$ term is equivalent to $\dot{\gamma}$ in the Casson equation. A weak-form Casson constitutive equation is derived as

$$
\mu\left(D_{\mathrm{II}}\right)=\frac{\left[\sqrt{\eta} \sqrt{\sqrt{2 D_{\mathrm{II}}}}+\sqrt{\tau_{\mathrm{y}}}\left(1-\mathrm{e}^{-n \sqrt{2 D_{\mathrm{II}}}}\right)\right]^{2}}{\sqrt{2 D_{\mathrm{II}}}} .
$$

The non-dimensionalized vorticity transport equation

Table 1. Blood yield stress values

\begin{tabular}{lll}
\hline $\begin{array}{l}\text { Hematocrit } \\
(\%)\end{array}$ & $\tau_{y}\left(\mathrm{dyn} \mathrm{cm}^{-2}\right)$ & Sources \\
\hline 46 & 0.05 & Cokelet et al. (1963) \\
Normal & 0.1089 & Charm and Kurland (1965) \\
Normal & $0.003-0.2$ & Charm and Kurland (1972) \\
42 & $0.144-0.256$ & Bate (1977) \\
& 0.1866 & Scott-Blair (1959) \\
\hline
\end{tabular}

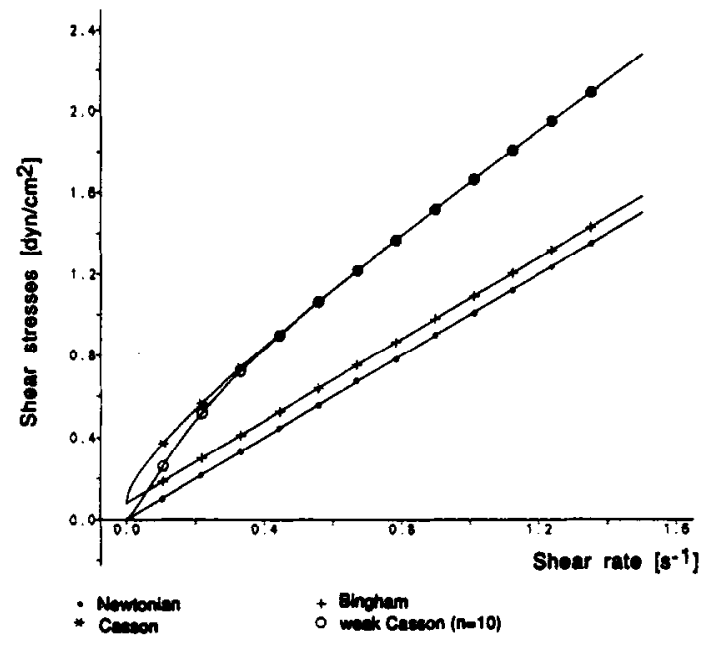

Fig. 3. Non-Newtonian models for a fluid with $\tau_{y}=0.1000 \mathrm{dyn} \mathrm{cm}^{-2}$. The weak Casson model is almost identical to the Casson model for shear rates exceeding $0.4 \mathrm{~s}^{-1}$.

is derived as

$$
\begin{aligned}
\omega_{t}+\psi_{y} \omega_{x}-\psi_{x} \omega_{y}= & \frac{1}{R e_{\mathrm{pt}}}\left[\left(\phi_{x x}-\phi_{y y}\right)\left(\psi_{y y}-\psi_{x x}\right)\right. \\
& -4 \phi_{x y} \psi_{x y}+2 \phi_{x} \omega_{x} \\
& \left.+2 \phi_{y} \omega_{y}+\phi \nabla^{2} \omega\right]
\end{aligned}
$$

Here $\omega$ is the vorticity, $\psi$ the stream function, $(x, y)$ the spatial domain, and $t$ the time. The variable $\phi$ is a dimensionless viscosity function defined as

$$
\phi=\frac{\mu}{\eta}=\frac{\left[\sqrt{\sqrt{2 D_{\mathrm{II}}}}+\sqrt{\bar{\tau}_{\mathrm{y}}}\left(1-\mathrm{e}^{-n \sqrt{2 D_{\mathrm{II}}}}\right)\right]^{2}}{\sqrt{2 D_{\mathrm{II}}}} .
$$

Here $\bar{\tau}_{\mathrm{y}}$ is a dimensionless yield shear stress defined as

$$
\tilde{\tau}_{\mathrm{y}}=\frac{L}{V} \frac{\tau_{\mathrm{y}}}{\eta}=\frac{\rho L^{2}}{R e_{\mathrm{pt}} \eta} \frac{\tau_{\mathrm{y}}}{\eta}=\frac{\tau_{y}^{\prime}}{R e_{\mathrm{pt}}}
$$

with

$$
\tau_{y}^{\prime}=\frac{\rho L^{2}}{\eta} \frac{\tau_{y}}{\eta} .
$$

The dimensionless shear stress is

$$
\tau=\left[\sqrt{\sqrt{2 D_{\mathrm{II}}}}+\sqrt{\bar{\tau}_{\mathrm{y}}}\left(1-\mathrm{e}^{-n \sqrt{2 D_{\mathrm{ut}}}}\right)\right]^{2} .
$$

The second invariant of the strain rate tensor is expressed in terms of the stream function as

$$
D_{\mathrm{II}}=2 \psi_{x y}^{2}+\frac{1}{2}\left(\psi_{y y}-\psi_{x x}\right)^{2} .
$$

An elliptic coordinate transformation system (Thompson et al., 1977) is simplified and adopted to deal with a complicated geometric shape. The vorticity transport equation (13) in the physical domain $(x, y)$ is transformed into the rectangular computa- 
tional domain $(\xi, \eta)$ as

$$
\begin{aligned}
& \omega_{t}+\left(\psi_{\eta} \omega_{\xi}-\psi_{\xi} \omega_{\eta}\right) / J \\
&= \frac{1}{R e_{\mathrm{p}} J^{2}}\left\{-\frac{1}{J^{2}}\left(\bar{\alpha} \phi_{\xi \xi}-2 \bar{\beta} \phi_{\xi \eta}+\bar{\gamma} \phi_{\eta \eta}+\bar{\sigma} \phi_{\eta}+\bar{\tau} \phi_{\xi}\right)\right. \\
&\left(\bar{\alpha} \psi_{\xi \xi}-2 \bar{\beta} \psi_{\xi \eta}+\bar{\gamma} \psi_{\eta \eta}+\bar{\sigma} \psi_{\eta}+\bar{\tau} \psi_{\xi}\right) \\
& \quad-\frac{4}{J^{2}}\left(\alpha^{\prime} \phi_{\xi \xi}-\beta^{\prime} \phi_{\xi \eta}+\gamma^{\prime} \phi_{\eta \eta}-\sigma^{\prime} \phi_{\eta}-\tau^{\prime} \phi_{\xi}\right) \\
&\left(\alpha^{\prime} \psi_{\xi \xi}-\beta^{\prime} \psi_{\xi \eta}+\gamma^{\prime} \psi_{\eta \eta}-\sigma^{\prime} \psi_{\eta}-\tau^{\prime} \psi_{\xi}\right) \\
& \quad+2\left[\left(\alpha \phi_{\xi}-\beta \phi_{\eta}\right) \omega_{\zeta}+\left(-\beta \phi_{\xi}+\gamma \phi_{\eta}\right) \omega_{\eta}\right] \\
&\left.\quad+\phi\left(\alpha \omega_{\xi \xi}-2 \beta \omega_{\xi \eta}+\gamma \omega_{\eta \eta}+\sigma \omega_{\eta}+\tau \omega_{\xi}\right)\right\}
\end{aligned}
$$

Here $J, \alpha, \beta, \gamma, \sigma$ and $\tau$ are the conventional coordinate transformation coefficients (Thompson et al., 1977), while $\bar{J}, \bar{\alpha}, \bar{\beta}, \bar{\gamma}, \bar{\sigma}, \bar{\tau}, J^{\prime}, \alpha^{\prime}, \beta^{\prime}, \gamma^{\prime}, \sigma^{\prime}$ and $\tau^{\prime}$ are additional coordinate transformation coefficients in the non-Newtonian model (Lou, 1990).

The Poisson equation is similar to that for a New. tonian fluid (Lou, 1990):

$\alpha \frac{\partial^{2} \psi}{\partial \xi^{2}}-2 \beta \frac{\partial^{2} \psi}{\partial \xi \partial \eta}+\gamma \frac{\partial^{2} \psi}{\partial \eta^{2}}+\sigma \frac{\partial \psi}{\partial \eta}+\tau \frac{\partial \psi}{\partial \xi}=-J^{2} \omega$.

The second invariant of the strain rate tensor is

$$
\begin{aligned}
D_{\mathrm{U}}= & \frac{2}{J^{4}}\left(\alpha^{\prime} \psi_{\xi \xi}-\beta^{\prime} \psi_{\zeta \eta}+\gamma^{\prime} \psi_{\eta \eta}-\sigma^{\prime} \psi_{\eta}-\tau^{\prime} \psi_{\xi}\right)^{2} \\
& +\frac{1}{2 J^{4}}\left(\bar{\alpha} \psi_{\xi \xi}-2 \bar{\beta} \psi_{\xi \eta}+\bar{\gamma} \psi_{\eta \eta}+\bar{\sigma} \psi_{\eta}+\bar{\tau} \psi_{\xi}\right)^{2} .
\end{aligned}
$$

Along the centerline of the trunk, where a potential flow is assumed, the second invariant is

$$
D_{\mathrm{Il}}=\frac{2}{J^{4}}\left(\alpha^{\prime} \psi_{\xi \xi}-\beta^{\prime} \psi_{\xi \eta}+\gamma^{\prime} \psi_{\eta \eta}-\sigma^{\prime} \psi_{\eta}-\tau^{\prime} \psi_{\xi}\right)^{2} .
$$

At the inlet and outlet, where a fully developed flow is assumed, and along solid walls, the second invariant is

$$
D_{\mathrm{II}}=\frac{1}{2} \omega^{2}
$$

No-slip conditions apply on the solid vessel walls. The stream function is constant because there is no flow across the wall. The vorticity is evaluated using the Woods' approximation (Woods, 1954), with second-order accuracy.

A potential flow region exists along the centerline of the trunk, along a constant $\eta$ line. The stream function is constant, and vorticity is zero because there is no flow across this line of symmetry.

The flow is fully developed at the inlet, on a constant $\xi$ line. The flow disturbance caused by the bifurcation is minimum at the inlet because of the convec. tive nature.

An experimentally measured, physically pulsatile blood flow rate proximal to the aortic bifurcation (Stettler et al., 1981) is used as the inlet flow rate (Fig. 2). The total period lasts for $1 \mathrm{~s}$ in a normal case. Similar flow wave forms have been observed (Holen- stein et al., 1980; Caro and Parker, 1987) and used in experimental studies (Walburn and Stein, 1982; Friedman et al., 1981, 1983; Rieu et al., 1985) and in a numerical study (O'Brien et al., 1976).

The stream function and vorticity at the inlet are obtained by numerically solving the simplified governing equations for a fully developed flow, with the given flow rate as a boundary condition (Lou, 1990).

The branch outlet is at another constant $\xi$ line with a fully developed flow. The vorticity and stream function values are not imposed at the outlet, where numerical reflection and disturbance may be induced. Soft boundary conditions (Roache, 1972) are used instead. The second derivative of the stream function and the first derivative of the vorticity are set to zero in the axial direction.

The finite difference method is used in the current study. The vorticity transport equation, as well as the Poisson equation, are solved with the successive-overrelaxation (SOR) method to overcome the strong non-linearity inherent in a non-Newtonian fluid. The time integration of the vorticity is performed implicitly to ensure stability. To save computing time, $\phi$ is evaluated explicitly, i.e. the second invariant is calculated using the stream function from the previous time step. All spatial derivatives at interior grid points in the governing equations are discretized by a central difference scheme. To avoid possible numerical instability and to increase accuracy, only the first derivatives of vorticity in the convection terms of the vorticity transport equation are treated by a hybrid upwind scheme.

When the yield shear stress is zero, steady and unsteady flows of a Newtonian fluid in an aortic bifurcation from the current computer program are identical to those from a Newtonian fluid program that was tested to be accurate (e.g. a wall vorticity error of $0.13 \%$ ) for an unsteady starting flow and for a cavity flow (Lou and Yang, 1991; Lou, 1990). The program is also tested for a fully developed flow of a Casson fluid in a two-dimensional channel. With $R e_{\mathrm{pt}}=1300$, the analytical solutions (Lou, 1990) for vorticity at the wall are 12.0, 12.392 and 12.540 for

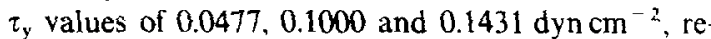
spectively. Their numerical counterparts, with $n=10$, are $11.984,12.393$ and 12.550 .

The computational mesh used for most of the cases in the present study has 41 nodes across the channel and 137 nodes along the axial direction (Fig. 4). It is non-uniform to give the best resolution around the hip and vertex, and near the walls. The convergence in vorticity with respect to the grid size is within $1 \%$ in both the axial and width directions (Lou, 1990).

\section{RESULTS}

Cases with various yield shear stresses and area ratios are studied (Table 2). They have some identical parameters: $R e_{\mathrm{pt}}=1300, \alpha=8.8623$ for 60 
beats $\left.\min ^{-1}\right), \beta=75^{\circ}, r_{\mathrm{i}}=0.15 L$ and $r_{\mathrm{o}}=0.2 L$. Cases $\mathrm{N} 0, \mathrm{~N} 1, \mathrm{~N} 2$ and N3 correspond to $\tau_{\mathrm{y}}$ of $0.0,0.0477$, 0.1000 and $0.1431 \mathrm{dyn} \mathrm{cm}^{-2}$, respectively. Case NO is



Fig. 4. Mesh for Cases N0, N1, N2 and N3 with $A R=0.75$, $\beta=75^{\circ}, L_{\mathrm{t}}=L, L_{\mathrm{b}}=4 L, r_{\mathrm{i}}=0.15 L, r_{\mathrm{o}}=0.2 L, 41$ nodes across the branch and 137 nodes along the axis. It is nonuniform to give the best resolution around the hip and vertex, and near the walls. Only the part of the mesh around the bifurcation is shown here, and the grid size grows larger towards the two ends.
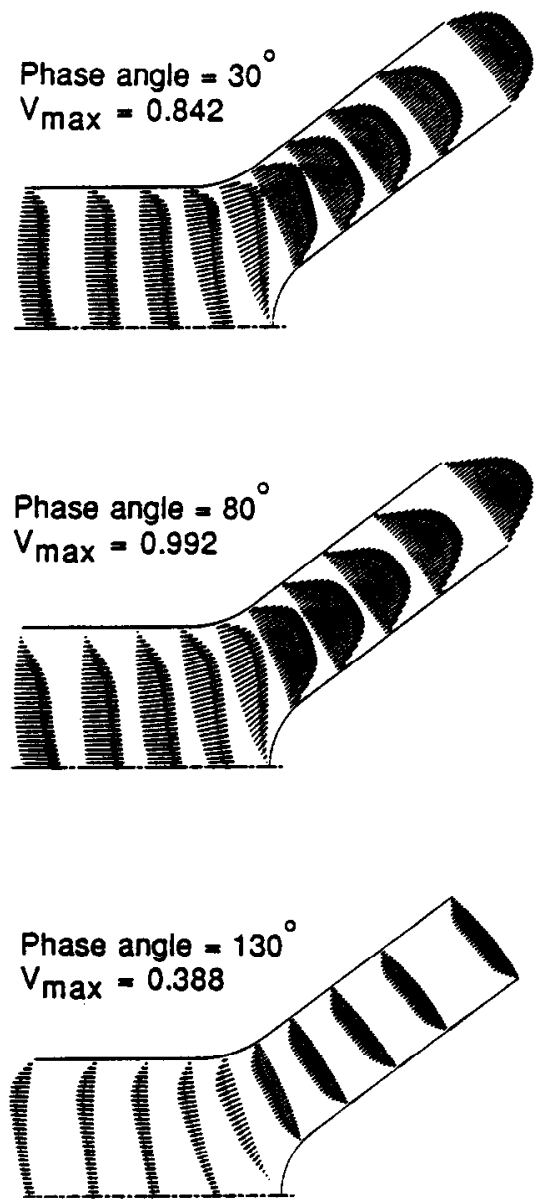

a limiting case and serves as a reference for comparing the effect of the non-Newtonian model.

In the interest of brevity, general results are presented only for Case N1 (Figs 5-8). The sign of the shear stress along the inner wall is reversed so that the shear stress is positive when the flow is forward, and negative when the stream is reversed. The $s$ for the inner wall is the distance from the vertex; the $s$ for the outer wall is the distance from the hip, positive downstream and negative upstream. $s$ is dimensionless and is measured along a curve instead of a direct distance.

Table 2. Cases with a non-Newtonian fluid and rigid walls

\begin{tabular}{lcl}
\hline Case no. & $\tau_{y}\left(\right.$ dyn cm $\left.^{-2}\right)$ & AR \\
\hline N0 & 0.0 & 0.75 \\
N1 & 0.0477 & 0.75 \\
N2 & 0.1000 & 0.75 \\
N3 & 0.1431 & 0.75 \\
N4 & 0.1000 & 1.1 \\
\hline
\end{tabular}
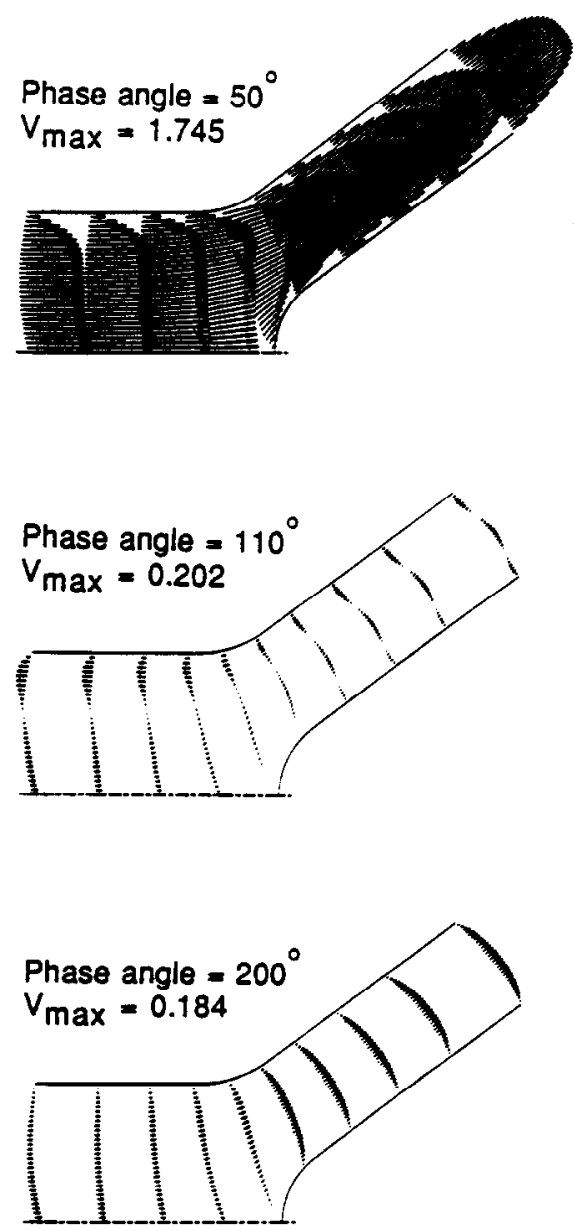

Fig. 5. Velocity profiles for Case N1 with $\tau_{\mathrm{y}}=0.0477 \mathrm{dyn} \mathrm{cm}^{-2}, R e_{\mathrm{pt}}=1300, \alpha=8.8623, \mathrm{AR}=0.75, \beta=75^{\circ}$, $r_{\mathrm{i}}=0.15 L$ and $r_{\mathrm{o}}=0.2 L$. Velocity profiles are flat everywhere except at the vertex, where a new boundary layer develops. 


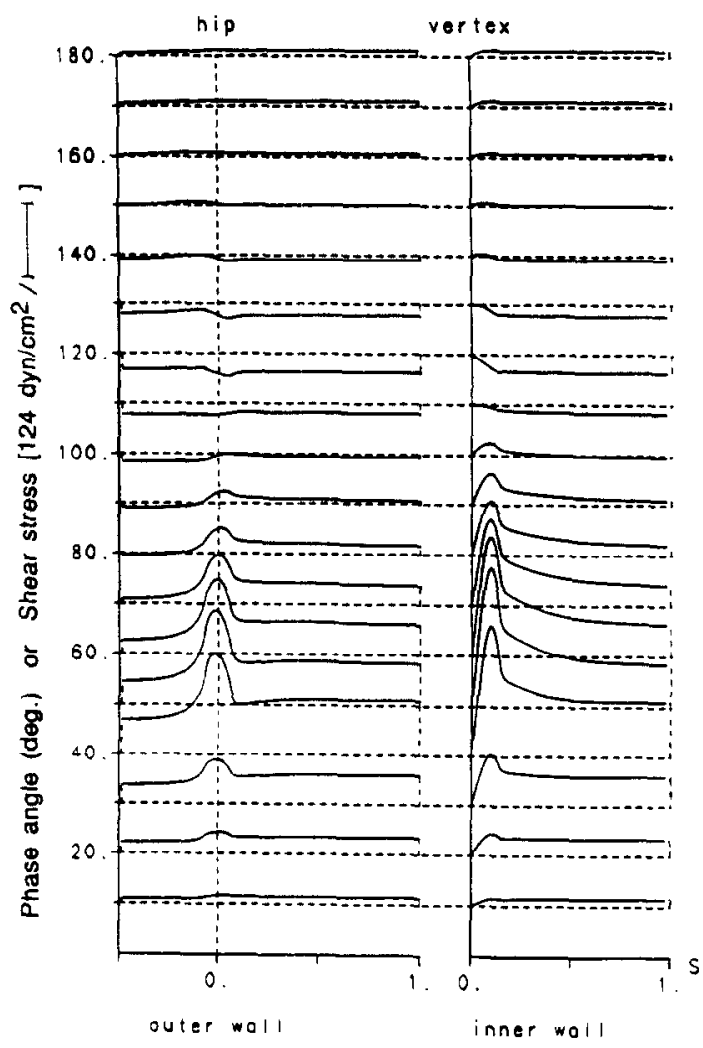

Fig. 6. Shear stress along the walls for Case N1 with $\tau_{y}=0.0477$ dyn cm $^{-2}, R e_{\mathrm{pt}}=1300, \alpha=8.8623, \quad \mathrm{AR}=0.75$, $\beta=75^{\circ}, r_{1}=0.15 L$ and $r_{0}=0.2 L$. The shear stress changes with time at all points except at the vertex, where the shear is always zero.
The overall patterns for a non-Newtonian fluid flow are similar to those for a Newtonian fluid flow, which have been presented and discussed in detail in a Newtonian fluid model study (Lou and Yang, 1991). They will be stated only briefly, while the emphasis is on the differences between the Newtonian and the non-Newtonian models.

No permanent eddies are found owing to the pulsatile nature of a physiological flow and the vertex wedge effect. A temporary eddy exists distal to the hip under certain conditions, for instance, a large area ratio. The tiny vertex point, a stagnation point, is the only place of a permanent low shear stress. There are two high shear stress points, one on the outer wall immediately distal to the hip and the other on the inner wall distal to the vertex. The latter generally has the highest shear stress because of the wedge effect of the vertex. A new number, called the wall shear stress concentration factor, is introduced to express the effect of bifurcation. It is defined as

$$
f=\frac{\text { peak wall shear stress }}{\text { wall shear stress at the trunk inlet }}
$$

based on the temporal maximum values over a cycle $f$ has a meaning similar to the stress concentration factor in solid mechanics. Depending upon the parameters, the shear stress concentration factor changes between 1.5 and 5.0, with the maximum shear stress at $280 \mathrm{dyn}^{-2}$.

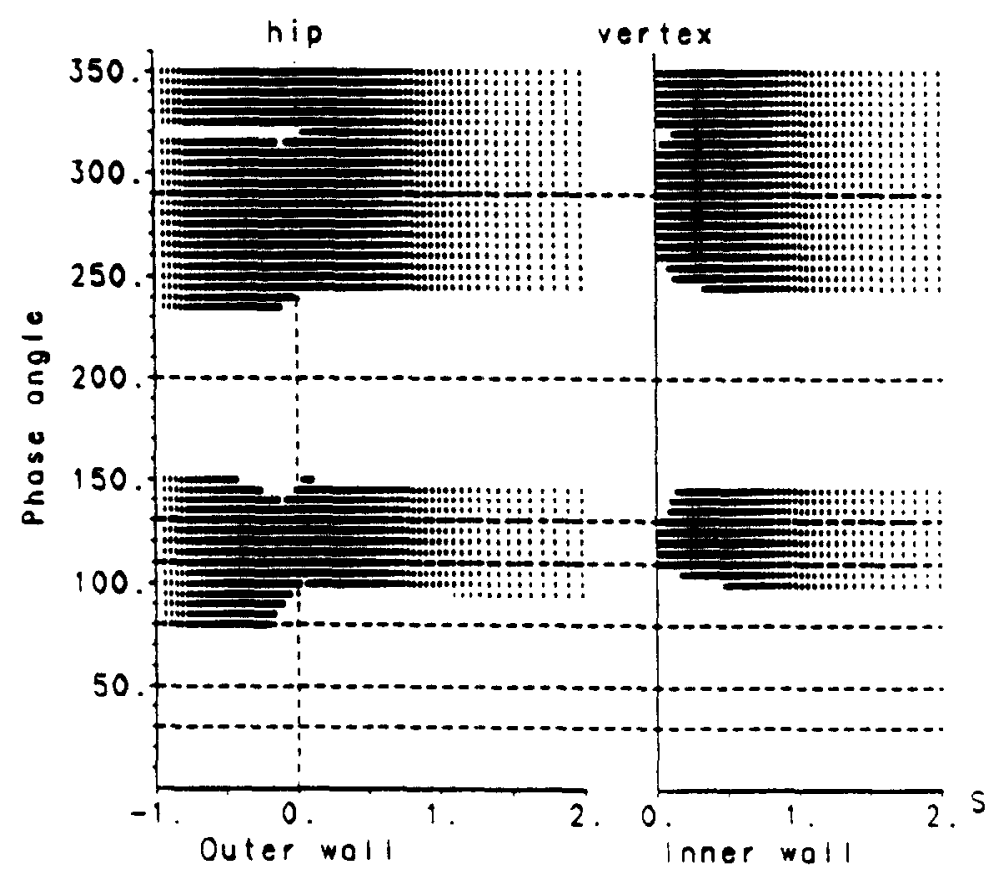

Fig. 7. Reverse flow patterns along the walls for Case N1 with $\tau_{y}=0.0477 \mathrm{dyn} \mathrm{cm}^{-2}, R e_{\mathrm{pt}}=1300$, $\alpha=8.8623, \mathrm{AR}=0.75, \beta=75^{\circ}, r_{\mathrm{i}}=0.15 L$ and $r_{\mathrm{o}}=0.2 \mathrm{~L}$. The dotted areas show reverse flows. Each point represents a computational node. Dots are clustered around the hip and the vertex because of a high concentration of nodes. The dotted lines indicate significant phase angles in a cycle. All points, except the vertex, experience flow reversal during certain periods of a cycle. No permanent eddies are found because of the pulsatile nature of a physiological flow and the vertex wedge effect. 


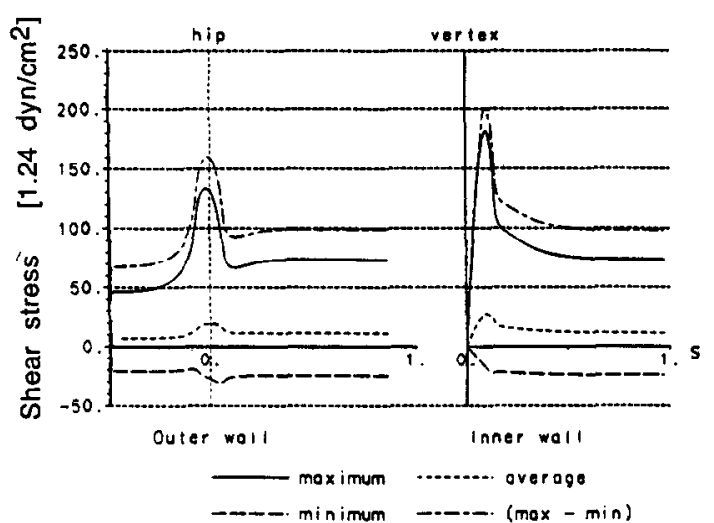

Fig. 8. Maximum, minimum, time-averaged absolute, and maximum - minimum shear stresses along the walls for Case N1. $\tau_{y}=0.0477$ dyn cm ${ }^{-2}, R e_{\mathrm{pt}}=1300, \alpha=8.8623, A R=0.75$, $\beta=75^{\circ}, r_{\mathrm{i}}=0.15 L$ and $r_{\mathrm{o}}=0.2 L$. The shear stress peaks proximal to the hip along the outer wall as well as distal to the vertex along the inner wall. The shear stress is permanently low only in a tiny region immediately surrounding the stagnant vertex.
The yield shear stress variation within a physiological range does not fundamentally change the velocity profiles. Flow reversal patterns do exhibit detectable differences (Fig. 9). For example, at a phase angle of $100^{\circ}$, the whole outer wall is covered by a reversal flow for a Newtonian fluid, while a tiny spot near the hip is exposed to the forward flow in non-Newtonian cases. The size of this spot is larger in a fluid with a higher $\tau_{y}$ value. The reappearance of the forward flow around a phase angle of $150^{\circ}$ is delayed as $\tau_{y}$ is increased. The forward flow in a Newtonian fluid prevails on both walls of the branch at a phase angle of $150^{\circ}$. In the case of a non-Newtonian fluid with $\tau_{y}=0.0477 \mathrm{dyn} \mathrm{cm}^{-2}$, reverse flow lingers near the hip on the outer wall. The residual reverse flow region is about three times larger for $\tau_{y}=0.1000 \mathrm{dyn}^{-2}$, and it covers the entire outer wall and part of the inner wall for $\tau_{y}=0.1431 \mathrm{dyn} \mathrm{cm}^{-2}$. At $320^{\circ}$, both walls are covered with the forward flow for the Newtonian fluid case, while only the outer wall in the trunk is covered with the forward flow for non-Newtonian fluids.

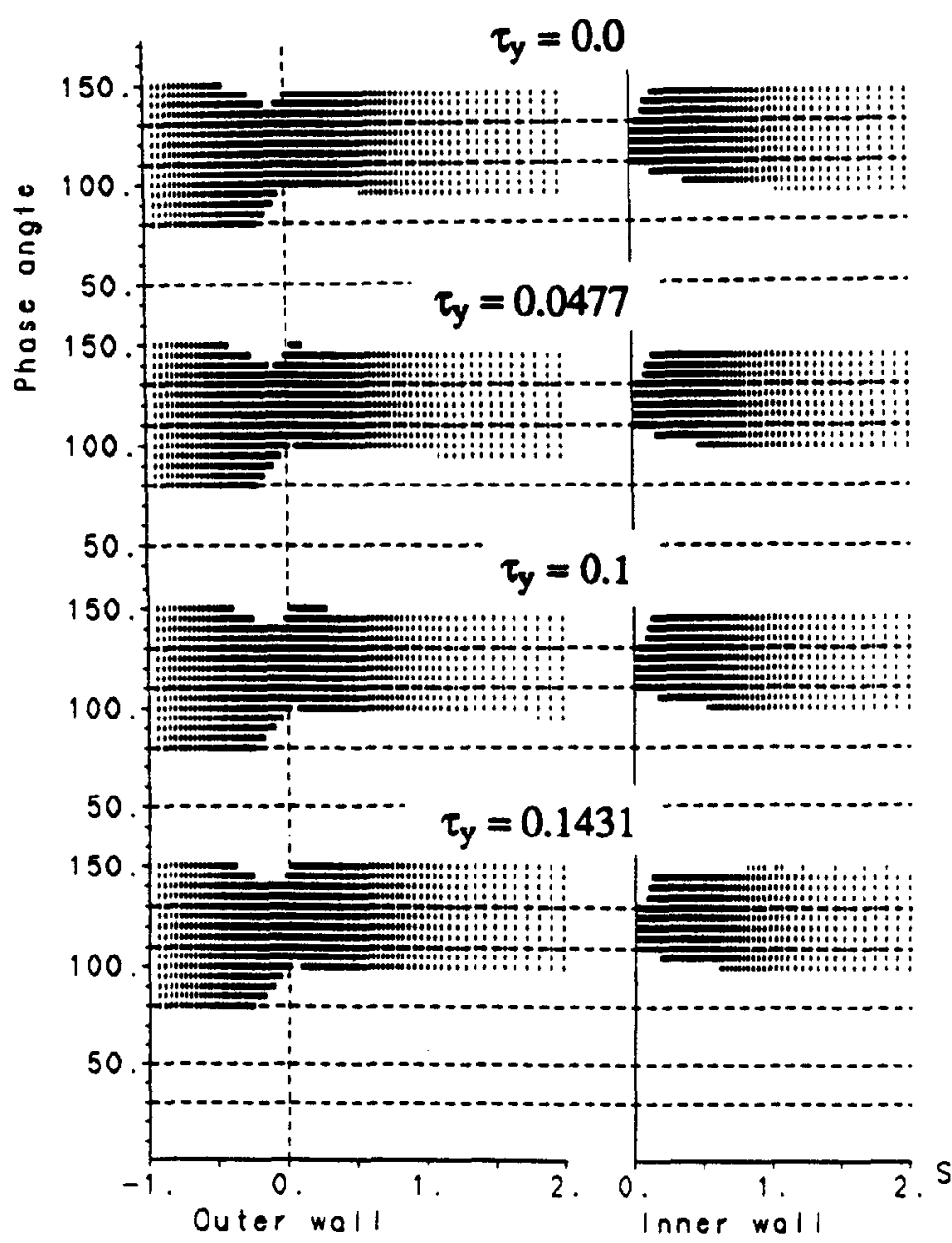

Fig. 9. Regions of reverse flow along the inner and outer walls versus phase angle for various $\tau_{y}$. The dotted areas show reverse flows. Each point represents a computational node. Dots are clustered around the hip and the vertex because of a high concentration of nodes. The dotted lines indicate significant phase angles in a cycle. A non-Newtonian fluid is more resistant to flow direction changes along the walls, either from a forward flow to a reverse one or vice versa. 
All the shear stresses increase with $\tau_{y}$ (Fig. 10) because the apparent viscosity rises with $\tau_{\mathrm{y}}$. The nonNewtonian effect is higher at the inlet and outlet than at the inner and outer peak points because the relative increase in viscosity is higher in a region of lower shear rates. As a result, the shear stress concentration factor falls from 4.06 to 3.93 for the inner wall, and from 2.97 to 2.87 for the outer wall, as $\tau_{y}$ changes from zero to $0.1431 \mathrm{dyn} \mathrm{cm}^{-2}$ (Fig. 11). Similarly, the relative increases in the time-averaged absolute shear stresses caused by the non-Newtonian behavior are much higher than those in the maximum shear stresses (Fig. 11). The deviation in the time-averaged absolute shear stress at the outlet, for example, is as high as $15.5 \%$.

Case N4 resembles Case 3 of the Newtonian model (Lou and Yang, 1991) except for its non-Newtonian behavior. The former shows $6.7,4.5$ and $5.4 \%$ higher temporal maximum shear stresses at the inlet, outer and inner wall peak points, respectively. These 4.5 and $5.4 \%$ increases at the wall peak spots are slightly higher than the 3.6 and $3.8 \%$ increases for Case N2 over Case N0 because the shear rate ranges near the wall peak points are lower in Cases $\mathrm{N} 4$ and 3 than in Cases N2 and N0.

\section{DISCUSSION}

The current study is intended to examine the possible effect of the non-Newtonian behavior of blood on hemodynamics that relate to atherogenesis. One of the assumptions implicit in the current model is that the arterial walls are rigid. However, the effect of wall

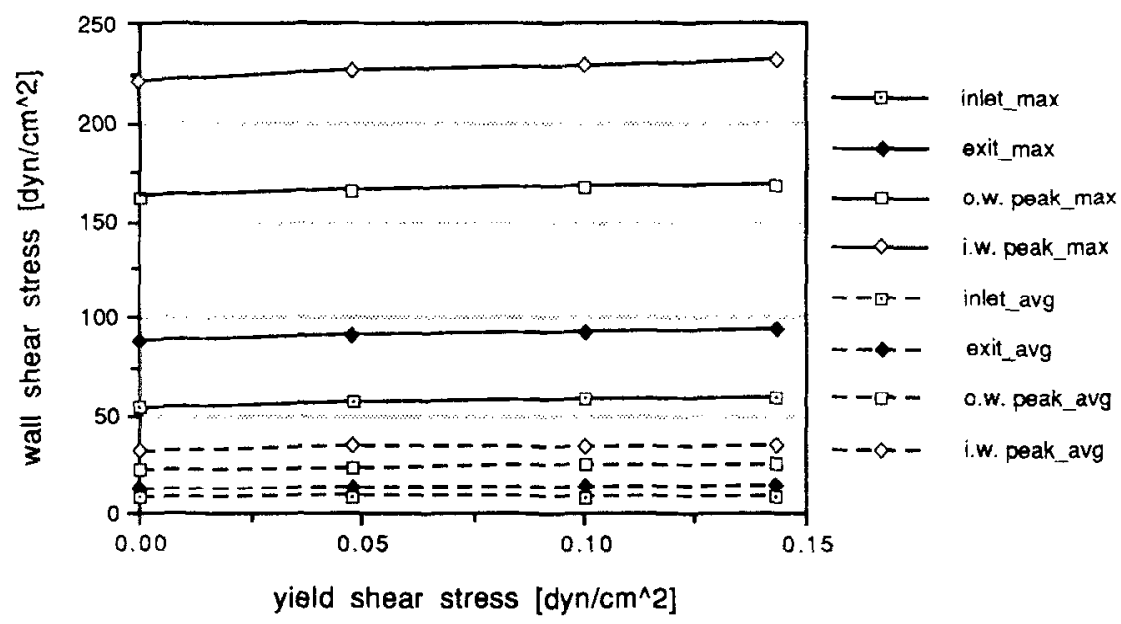

Fig. 10. Effect of $\tau_{y}$ on the wall shear stress (i.w.: inner wall; o.w.: outer wall; max: maaximum over a cycle; and avg: time-averaged absolute over a cycle). All the shear stresses increase with an increase in the yield shear stress $\tau_{y}$.

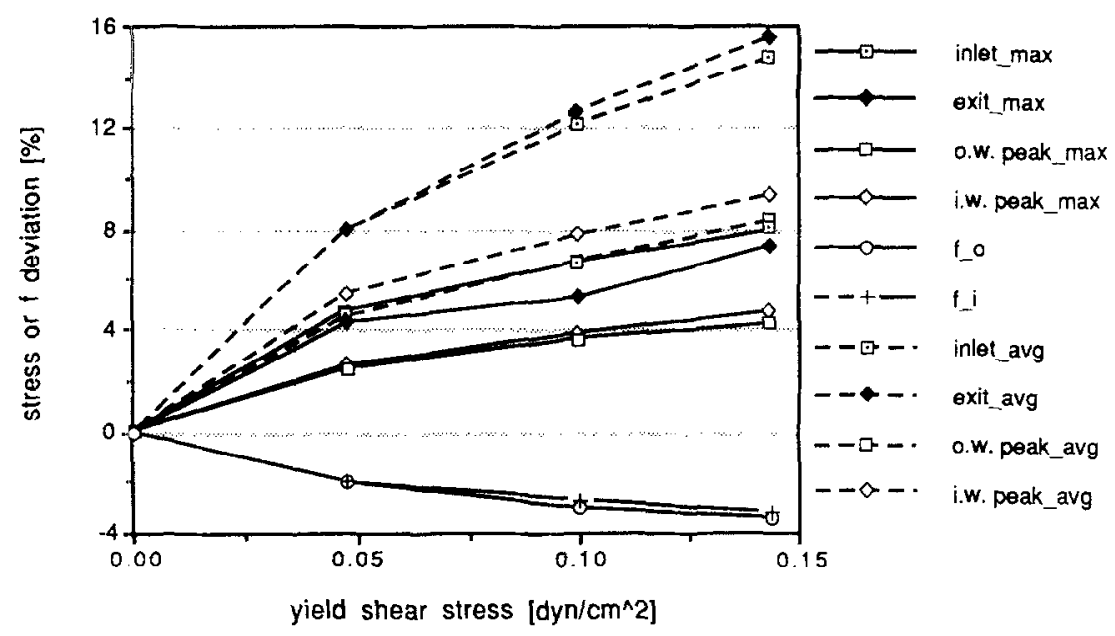

Fig. 11. Deviations of wall shear stress or the shear stress concentration factor from Newtonian behavior (i.w.: inner wall; $0 . w$.: outer wall; max: maximum over a cycle; and avg: time-averaged absolute over a cycle). The relative change induced by non-Newtonian rheology is higher in a region of lower shear rates (inlet and exit). Similarly, the relative increases in the time-averaged absolute shear stresses are much higher than those in the timewise maximum ones. 
flexibility on the maximum shear stress is about $10 \%$ (Lou, 1990). The geometry of the bifurcation is idealized to be two-dimensional, which does not cause large errors, as discussed in the introduction. One added advantage of a two-dimensional model is the facility with which a finer mesh can be employed, which is needed for an accurate evaluation of the wall shear stresses around the vertex and near the walls. A three-dimensional model with a fine mesh, of course, should be the ultimate goal.

Permanent eddies and regions of a significantly low time-averaged absolute shear stress do not exist around the aortic bifurcation as they do around the carotid bifurcation. These results agree qualitatively with the experimental observations (Walburn and Stein, 1980; Fukushima et al., 1988). In fact, the timeaveraged absolute shear stress at the hip is higher than the asymptotic values both in the trunk and the branch (Fig. 8). However, it is difficult to compare the results from the current study with the results from the three-dimensional models mentioned in the introduction, which have either been applied to locations other than the aortic bifurcation, or have used parameters that deviate greatly from the physiological ones. For example, Yung et al. (1990) found a large, permanent region of reversed flow primarily because of the non-physiologically large area ratio of 2 and the steady flow used in the model.

The maximum shear stress from the current and the previous (Lou and Yang, 1991) studies is about

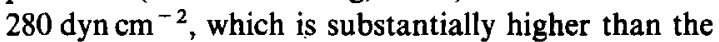
$64 \mathrm{dyn} \mathrm{cm}^{-2}$ from Friedman et al. (1981). However, Friedman et al. (1981) obtained $64 \mathrm{dyn}^{-2}$ along the inner wall about 0.87 branch width distal to the vertex. In the case that is closest to that of Friedman et al. (1981), case 1d in Lou and Yang (1991) and Lou (1990) with $\mathrm{AR}=1.0$ and $R e_{\mathrm{pt}}=1600$, the shear stress peaks to $220 \mathrm{dyn} \mathrm{cm}^{-2}$ along the inner wall about 0.4 branch width distal to the vertex. The temporal maximum shear stress at a point 0.87 branch width distal to the vertex is about $110 \mathrm{dyn} \mathrm{cm}^{-2}$, which is still higher than that from Friedman et al. (1981). Friedman et al. (1981) did not directly measure the shear stress at the wall. Longitudinal velocities at site 12 were measured with the laser Doppler velocimeter, and the shear rates were extrapolated by a linear curve-fitting method. The site was $0.058 \mathrm{~cm}$ vertical distance away from the inner wall surface of a branch of a diameter of $1.0 \mathrm{~cm}$. The boundary layer thickness of a physiologically pulsatile flow around the aortic bifurcation is about $0.1 \mathrm{~cm}$ or less. Linear curve-fitting with the data point at $0.058 \mathrm{~cm}$ may underestimate the shear rate, even with accurate velocity and position data.

Similarly, Perktold et al. (1991b) found that their three-dimensional numerical results for a carotid bifurcation have consistently higher shear stress than those of the experimental results from Ku et al. (1985), who used a method similar to that by Friedman et al. (1981). In another study, Brech and Bellhouse (1973) found that the directly measured (with thin-film heated elements) wall shear stress is always greater than that taken from the velocity plots. For a pulsatile flow with $R e_{\mathrm{pt}}=1500$ and $\alpha=8.776$ (according to the standard definition used for a circular tube) in an aortic bifurcation with $\beta=90^{\circ}$ and $\mathrm{AR}=1.12$, the peak maximum shear stress extrapolated from the directly measured data is about $310 \mathrm{dyn} \mathrm{cm}^{-2}$. This value is slightly higher than those of the current study.

With the above discussion in mind, the shear stress values and main features of the flow pattern from the current model are reasonable.

No fundamental diffecnce between the flow patterns of Newtonian and non-Newtonian fluid models is found in the current study. Using the Casson model, Perktold et al. $(1989,1991$ a) came to a similar conclusion in a three-dimensional numerical study of a cerebrovascular bifurcation with an aneurysm and a carotid bifurcation. Liepsch and coworkers (Liepsch, 1985, 1986; Liepsch and McMillan, 1986; Liepsch and Moravec, 1984; Moravec and Liepsch, 1983) found much more significant non-Newtonian effects in T-shaped bifurcation experiments. However, they used a power-law-non-Newtonian fluid. The current study does find some subtle differences between Newtonian and non-Newtonian fluids.

The current study reveals that a non-Newtonian fluid is more resistant to flow changes, from a forward flow to a reverse flow or vice versa. The reason is that a non-Newtonian fluid has a higher apparent viscosity than a Newtonian fluid within the same range of shear rates. Since the limiting apparent viscosity is used in calculating the Womersley number, a higher apparent viscosity implies a lower Womersley number for a non-Newtonian fluid, resulting in a more viscous resistance to the flow, and less phase difference for boundary layers and the center stream. The above observation is consistent with the findings of other investigators. A stenosis study with a steady biviscosity fluid flow (Nakamura and Sawada, 1988) revealed that the non-Newtonian property of blood diminishes the distortion of the flow pattern, flow separations and overshoot in certain segments of the velocity profile. Another stenosis study with an unsteady biviscosity fluid flow (Nakamura and Sawada, 1990) found that the non-Newtonian property of blood tends to restrain the occurrence and growth of flow separation in an accelerating flow, and promotes the disappearance of flow separation in decelerating flow. Inside an aneurysm at a cerebral bifurcation, slightly more circulation occurs with a Newtonian fluid than with a Casson fluid (Perktold et al., 1989). A similar smoothing effect on the velocity profiles was detected in T-shaped bifurcation studies (Liepsch, 1985; Moravec and Liepsch, 1983). It was disclosed that power-law non-Newtonian fluids experience an extra flow separation zone distal to the outlet, a possible feature of a T-shaped bifurcation or the power-law fluid.

With the asymptotic apparent viscosity in the Casson fluid equal to the viscosity of a Newtonian fluid, it is found that the wall shear stresses in a non-New- 
tonian model are generally higher than those in a Newtonian counterpart. Similar shear stress increases in a Casson fluid were found in a stenosis study using a perturbation method (Chaturani and Samy, 1986), and wall shear stress increases in a stenosis were disclosed using a couple stress fluid model (Srivastava, 1985).

A study on a two-dimensional conduit expansion of power-law fluids (Giaquinta and Hung, 1968) revealed that a departure from Newtonian behavior diminishes with an increase in the Reynolds number, equivalent to a rise in the shear rate, which is consistent with the results from the current study. The velocity and flow pattern deviations between a Newtonian fluid and a Casson fluid are more appreciable at the minimum flow rate than at the maximum flow rate in a cerebral bifurcation with an aneurysm (Perktold et al, 1989).

In a stenosis study using both the power-law and Casson fluids, Shukla et al. (1980) found that two parameters that are equivalent to the shear stress concentration factor in this study decrease as $\tau_{y}$ in creases, in agreement with the results from the present study (Fig. 11).

There have been two main hypotheses that relate to a possible hemodynamic role in atherogenesis: the low shear stress (Caro et al, 1971) and the high shear stress (Fry, 1968). A continuous exposure to a shear stress of $380 \mathrm{dyn} \mathrm{cm}^{-2}$ can cause degradation in canine walls within $1 \mathrm{~h}$ (Fry, 1968). The temporal maximum shear stress from the current and the previous (Lou and Yang, 1991) studies varies from 63 to $279 \mathrm{dyn}^{-2}$, depending on the geometric and flow

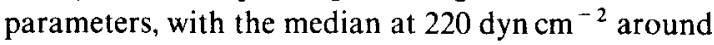
the flow divider

The low shear stress theory postulates relatively complicated bio-mass transfers, inhibited by low wall shear rates and recirculations (Caro et al., 1971). This theory is the prevailing one at the moment, although it is slightly dangerous to believe one or the other totally (Nerem and Levesque, 1987). The correlation between high atherosclerosis probability areas and expected low wall shear stress regions is far from perfect (Cornhill et al., 1990, 1985). Many other localizing biochernical, cellular, mass transport, and structural factors may be involved.

The outer wall intima is thicker than the apical intima at the aortic bifurcation, except in subjects younger than one month (Yoshida et al., 1988), and the vertex is spared of atherosclerotic lesions (Cornhill and Herderick 1988). However, not every bifurcation has a low shear region along the outer wall as expected by Yoshida et al. (1988) and many others. Although the low shear and recirculation are evident along the outer walls of the carotid bifurcation, this and other studies with reasonable parameters do not show any permanent eddies at the aortic bifurcation. The time-averaged absolute shear rate, which is relevant to any mass transfer when there are no permanent eddies, at the point slightly distal to the hip on the outer wall changes from 4 to $14 \mathrm{dyn}^{-2}$, which is comparable to the asymptotic values in both the branch and the trunk. There is a point on the outer wall, close to the hip, where the maximum shear stress changes from 51 to $218 \mathrm{dyn} \mathrm{cm}^{-2}$, while its time-averaged absolute value changes from 7 to $30 \mathrm{dyn} \mathrm{cm}^{-2}$ (Lou, 1990). Only at a tiny tip of the flow divider can one expect a permanent low or zero shear stress.

The change of shear stress direction within a cardiac cycle or its amplitude has also been suggested to be related to atherogenesis, which, however, does not favor any particular point at the aortic bifurcation (Fig. 8). More recently, Thubrikar et al. (1990) proposed that the intramural stress concentration is related to atherosclerosis.

The effect of the non-Newtonian behavior of blood, at least that characterized by the Casson model, will be only quantitative around the aortic bifurcation if the shear stress per se is ever involved. The role of hemodynamics in atherogenesis is complex and incompletely understood. This investigation contributes information on the effect of the non-Newtonian nature of blood on the flow at the aortic bifurcation. It adds, therefore, to the data based on which a future clarification of the role of the hemodynamics may be possible. The non-Newtonian property of blood that possibly induces changes like clustering of blood cells may be involved in atherogenesis in other ways, e.g. influencing biomass transfer mechanisms.

Acknowledgements - The authors greatly appreciate sugges tions and advice from Drs William P. Graebel, Noboru Kikuchi, Tasos C. Papanastasiou, Gretar Tryggvason and Paul D. Stein.

\section{REFERENCES}

Bate, H. (1977) Blood viscosity at different shear rates in capillary tubes. Biorheology 14, 267-275.

Brech, R. and Bellhouse, B. J. (1973) Flow in branching vessels. Cardiovasc. Res. 7, 593600

Caro, C. G., Fitz-Gerald, J. M. and Schroter, R. C. (1971) Atheroma and arterial wall shear: observation, correlation and proposal of a shear dependent mass transfer mechanism for atherogenesis. Proc. R. Soc. Lond. B177, $109-159$.

Caro, C. G. and Parker, K. H. (1987) The effect of haemodynamic factors on the arterial wall. In Atherosclerosis-Biology and Clinical Science (Edited by Olsson, A. G.), pp. 183-195. Churchill Livingstone, Edinburgh.

Casson, N. (1959) A flow equation for the pigment oil suspensions of the printing ink type, In Rheology of Disperse System (Edited by Mill, D.C.), pp. 84-102. Pergamon Press, New York.

Charm, S. E. and Kurland, G. S. (1962) The flow behavior and shear-stress shear rate characteristic of canine blood. Am. J. Physiol. 203, 417-421.

Charm, S. E. and Kurland, G. S. (1965) Viscometry of human blood for shear rates of $0-100,0001 / \mathrm{sec}$. Nature (London) 206, $617-618$.

Charm, S. E. and Kurland, G. S. (1972) Blood rheology. In Cardiovascular Fluid Dynamics, Vol. 2 (Edited by Bergel, D. H.), Ch. 15, pp. 157-203. Academic Press, London.

Chaturani, P. and Samy, R. P. (1986) Pulsatile flow of Cas son's fluid through stenosed arteries with applications to blood flow. Biorheology 23, 499-511.

Cokelet, G. R, Mcrill, E. W., Gilliland. E, R.. Shin. H. Britter, A. and Wells, R. E. (1963) Rheology of human 
blood: measurement near and at zero rate. Trans. Soc. Rheol. 7, 303-317.

Cornhill, J. F., Barrett, W. A., Herderick, E. E., Mahley, R. W. and Fry, D. L. (1985) Topographic study of sudanophilic lesions in cholesterol-fed minipigs by image analysis. Arteriosclerosis 5, 415-26.

Cornhill, J. F. and Herderick, E. E. (1988) Localization of human atherosclerotic lesions. In Proceedings of the World Congress on Medical Physics and Biomedical Engineering. San Antonio, Texas; in Phys. Med. Biol. 33 (Suppl. 1), 251.

Cornhil, J. E., Herderick, E. E. and Stary, H. C. (1990) Topography of human aortic sudanophilic lesions. Monogr. Atheroscler. 15, 13-19.

Feuerstein, A. I., El Masry, O. A. and Round, G. F. (1976) Arterial bifurcation flows - effects of flow rate and area ratio. Can. J. Physiol. Pharmacol. 54, 795-808.

Frasher, W. G., Wayland, H. and Meiselman, H. J. (1968) Viscometry of circulating blood in dogs. I. Heparin injection, II. Platelet removal. J. appl. Physiol. 25, 751-760.

Friedman, M. H., Hutchins, G. M., Bargeron, C. B., Deters, O. J. and Mark, F. F. (1981) Correlation between intimal thickness and fluid shear in human arteries. Atherosclerosis 39, 425-436.

Friedman, M. H., Deters, O. J., Marks, F. F., Bargeron, C. B. and Hutchins, G. M. (1983) Arterial geometry affects hemodynamics. Atherosclerosis 46, 225-231.

Fry, D. L. (1968) Acute vascular endothelial changes associated with increased blood velocity gradients. Circ. Res. 22, 165-197.

Fukushima, T., Homma, T., Harakawa, K., Sakata, N. and Azuma, T. (1988) Vortex generation in pulsatile flow through arterial bifurcation models including the human carotid artery. J. biomech. Engng 110, 166-171.

Fung, Y. C. (1981) Biomechanics-Mechanical Properties of Living Tissues. Springer, New York.

Giaquinta, A. R. and Hung, T. K. (1968) Slow non-Newtonian flow in a zone of separation. J. Engng Mech. Div. ASCE 94, No. EM6, Proc. Paper 6319, pp. 1521-1538.

Goldman, A. (1981) Two-dimensional and axisymmetric bifurcated channel flow. $A I A A J .19,1274-1275$.

Hearshen, D. O. (1991) Comparison of velocity encoded magnetic resonance imaging and fluid dynamic simulation in symmetrically branched tubes. Personal communication.

Holenstein, R., Niederer, P. and Anliker, M. (1980) A viscoelastic model for use in predicting arterial pulse waves, J. biomech. Engng 102, 318-325.

Hussian, A. K. M. F. (1977) Mechanics of pulsatile flows of relevance to the cardiovascular system. In Cardiovascular Flow Dynamics and Measurements (Edited by Hwang, N. H. C. and Normann, N. A.), Ch. 15, pp. 541-632. University Park Press, Baltimore.

Ku, D. N., Giddens, D. P., Zarins, C. Z. and Glagov, S. (1985) Pulsatile flow and atherosclerosis in the human carotid bifurcation. Arteriosclerosis 5, 293-302.

Liepsch, D. (1985) Flow patterns in elastic models of branched tubes. Physicochem. Hydrodyn. 6, 699-701.

Liepsch, D. (1986) Velocity measurements of viscoelastic fluids in a $90^{\circ}$ bifurcation of a tube with rectangular cross-section. Physicochem. Hydrodyn. 7, 45-54.

Liepsch, D. W. and McMillan, D. E (1986) Laser-Doppler velocity measurements at a $90^{\circ}$ bifurcation with Newtonian and non-Newtonian Fluids. In Proceedings of Sixth International Congress Biorheology (Abstracts). Biorheology 23, 221.

Liepsch, D. and Moravec, S. (1984) Pulsatile flow on nonNewtonian fluid in distensible models of human arteries. Biorheology 21, 571-586.

Lightfoot, E. N. (1974) Transport Phenomena and Living Systems. Wiley, New York.

Lou, Z. (1990) A computer simulation of the flow field at the aortic bifurcation. Ph.D. thesis, University of Michigan, Ann Arbor.

Lou, Z. and Yang, W. J. (1991) A computer simulation of the blood flow at the aortic bifurcation. Biomed. Mater. Engng 1, 173-193.

Merril, E. W., Cokelet, G. R., Britten, A. and Wells, R. E. (1964) Rheology of human blood and the red cell plasma membrane. Bibl. Anat. 4, 51-57.

Merril, E. W., Margetts, W. G., Cokelet, G. R. and Gilliland, E. W. (1965) The Casson equation and rheology of blood near zero shear. In Symposium on Biorheology (Edited by Copley, A.), pp. 135-143. Interscience, New York.

Merril, E. W., Gilliland, E. R., Lee, T. S. and Salzman, E. W (1966) Blood rheology-effect of fibrinogen deduced by addition. Circ. Res. 18, 437.

Moravec, S. and Liepsch, D. (1983) Flow investigations in a model of a three-dimensional human artery with Newtonian and non-Newtonian fluids. Part I. Biorheology 20, 745-759.

Murray, C. D. (1926) The physiological principle of minimum work applied to the angle of branching or arteries. J. Gen. Physiol. 9, 835-841.

Nakamura, M. and Sawada, T. (1988) Numerical study on the flow of a non-Newtonian fluid through an axisymmetric stenosis. J. biomech. Engng 110, 137-143.

Nakamura, M. and Sawada, T. (1990) Numerical study on the unsteady flow of non-Newtonian fluid. $J$. biomech. Engng 112, 100-103.

Nerem, R. M. and Levesque, M. J. (1987) Fluid mechanics in atherosclerosis. In Handbook of Bioengineering (Edited by Skalak, R. and Chien, S.), pp. 21.1-21.22. McGraw-Hill, New York.

O'Brien, V., Ehrlich, L. W. and Friedman, M. H. (1976) Unsteady flow in a branch. J. Fluid Mech. 75, 315-336.

Papanastasiou, T. C. (1987) Flows of materials with yield. J. Rheol. 31, 385-404.

Patil, M. K. and Subbaraj, K. (1988) Finite element analysis of two dimensional steady flow in model arterial bifurcation. J. Biomechanics 21, 219-233.

Perktold, K., Peter, R. and Resch, M. (1989) Pulsatile nonNewtonian blood flow simulation through a bifurcation with an aneurysm. Biorheology 26, 1011-1030.

Perktold, K. and Peter, R. (1990) Numerical 3D-simulation of pulsatile wall shear stress in an arterial T-bifurcation model. J. biomed. Engng 12, 2-12.

Perktold, K., Resch, M. and Florian, H. (1991a) Pulsatile non-Newtonian flow characteristics in a three-dimensional human carotid bifurcation model. J. biomech. Engng 113, 464-475.

Perktold, K., Resch, M. and Peter, R. O. (1991b) Threedimensional numerical analysis of pulsatile flow and wall shear stress in the carotid artery bifurcation. J. Biomechanics 24, 409-420.

Rieu, R., Friggi, A. and Pelissier, R. (1985) Velocity distribution along an elastic model of human arterial tree. J. Biomechanics 18, 703-715.

Rindt, C. C. M., van Steenhoven, A. A., Janssen, J. D., Reneman, R. S. and Segal, A. (1990) A numerical analysis of steady flow in a three-dimensional model of the carotid artery bifurcation. J. Biomechanics 23, 461-473.

Roache, P. J. (1972) Computational Fluid Dynamics. Hermosa, Albuquerque, NM.

Scott-Blair, G. W. (1959) An equation of the flow of blood plasm and serum through glass capillaries. Nature 1983, 613-614.

Shukla, J. B., Parihar, R. S. and Rao, B. R. P. (1980) Effects of stenosis on non-Newtonian flow of the blood in an artery. Bull. Math. Biol. 42, 283-294.

Srivastava, L. M. (1985) Flow of couple stress fluid through stenotic blood vessels. J. Biomechanics 18, 479-485.

Stettler, J. C., Niederer, P., Anliker, M. and Casty, M. (1981) Theoretical analysis of arterial hemodynamics including 
the influence of bifurcations, Part II-critical evaluation of theoretical model and comparison with noninvasive measurements of flow patterns in normal and pathological cases. Ann. biomed. Engng 9, 165-175.

Thompson, J. F., Thames, F. C. and Mastin, C. W. (1977) Boundary-fitted curvilinear coordinate systems for solution of partial differential equations on fields containing any number of arbitrary two-dimensional bodies. NASA CR-2729, July.

Thubrikar, M. J., Roskelley, S. K. and Eppink, R. T. (1990) Study of stress concentration in the walls of the bovine coronary arterial branch. $J$. Biomechanics $23,15-26$.

van de Vosse, F. N., van Steenhoven, A. A., Janssen, J. D. and Reneman, R. S. (1990) A two-dimensional numerical analysis of unsteady flow in the carotid artery bifurcation: a comparison with a 3-D in vitro measurements and the influence of minor stenosis. Biorheology 27, 163-189.

Walburn, F. J. and Stein, P. D. (1980) Flow characteristics in symmetrically branched tubes simulating the human aortic bifurcation. J. biomech. Engng 102, 340-342.

Walburn, F. J. and Stein, P. D. (1982) A comparison of steady and pulsatile flow in symmetrically branched tubes. J. biomech. Engng 104, 66-68.

Walburn, F. J., Blick, E. F. and Stein, P. D. (1979) Effect of the branch-to-trunk ratio on the transition to turbulent flow: implications in the cardiovascular system. Biorheology 16, 411-417.

Wille, S. O. (1984) Numerical simulations of steady flow inside a three dimensional aortic bifurcation model J. biomed. Engng 6, 49-55.

Woods, L. C. (1954) A note on the numerical solution of fourth order differential equations. Aeronaut. Quart. 5(3), 176.

Yoshida, Y., Oyama, T., Yamane, T., Mitsumata, M. Yamaguchi, T. and Ooneda, G. (1988) Microarchitectural changes of human aortic bifurcations with reference to atherogenesis. In Proceedings of the World Congress on Medical Physics and Biomed. Eng. San Antonio. Texas; in Phys. Med. Biol. 33 (Suppl. 1), 250

Yung, C. N., de Witt, K. J. and Keith. Jr. T. G. (1990) Three-dimensional steady flow through a bifurcation. J. biomech. Engng 112, 189-197. 